\title{
Dry etching and sputtering
}

\author{
By C. D. W. WILKINSON AND M. RAHMAN ${ }^{2}$ \\ ${ }^{1}$ Department of Electronics and Electrical Engineering and \\ ${ }^{2}$ Department of Physics and Astronomy, University of Glasgow, \\ Glasgow G12 9UY, UK (c.wilkinson@elec.gla.ac.uk) \\ Published online 25 November 2003
}

Dry etching is an important process for micro- and nanofabrication. Sputtering effects can arise in two contexts within a dry-etch process. Incoming ions cause removal of volatile products that arise from the interaction between the dry-etch plasma and the surface to be etched. Also, the momentum transfer of an incoming ion can cause direct removal of the material to be etched, which is undesirable as it can cause electrical or optical damage to the underlying material. This is largely avoided in dry-etch processes by use of reactive chemistries, although in some processes this component of the etching can be significant. Etch processes, both machine type and possible etch chemistries, are reviewed. Methods of characterizing the electrical and optical damage related to ion impact at the substrate are described. The use of highly reactive chemistries and molecular constituents within the plasma is best for reducing the effects of damage.

Keywords: dry etching; damage; low-energy ions; reactive-ion etching; nanofabrication

\section{Introduction}

In the manufacture of semiconducting electronic and optoelectronic devices, a vital step is that of pattern transfer. Here the pattern that has been defined in a radiationsensitive material (the resist) is transferred into the relevant layer of material. As figure 1 shows, the transfer can be additive or subtractive; in practice subtractive processes are preferred as they have higher reliability and so higher yield.

Subtractive processing involves etching or the removal of material. This can be done using suitable wet chemicals or by dry etching in a vacuum system with the assistance of ions formed by an electrical discharge in a gas. Wet-chemical methods have a number of disadvantages. Wet etching is, in most cases, isotropic. The etched feature has curved walls and its width differs from that of the opening in the resist. If the aspect ratio (depth/width) of the desired feature is small, the isotopic nature of the etching is often not important; however, in the closely packed structures found in Very Large Scale Integration integrated circuits it is not acceptable. After wetchemical etching, the disposal of the partly used reagent can raise environmental issues. Monolayer-thick layers of hydrocarbons can inhibit wet etching. In situ control of etch depth is difficult.

One contribution of 11 to a Theme 'Sputtering: past, present and future. W. R. Grove 150th Anniversary Issue'. 


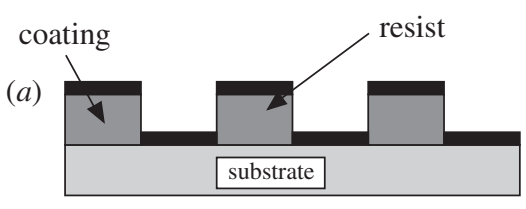

material to be etched

(c)

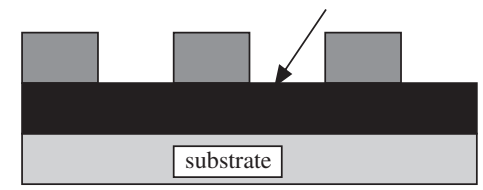

(b)

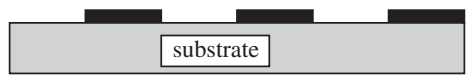

(d)

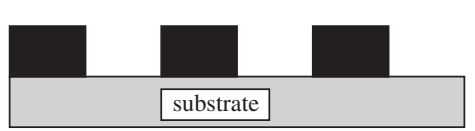

Figure 1. Distinction between additive and subtractive processing. (a) Additive processing; (b) after removal of resist in strong solvent; $(c)$ subtractive processing; $(d)$ after etching and removal of resist.

Dry etching relieves most of these difficulties (but at an increased capital cost). As ions can be directed by an electrical field, it is possible to gain some control over the profile of the etched feature. Often the composition of the gas mixture used in the discharge is chosen so that volatile products of the reaction with materials are formed that can be pumped away from the vacuum system. The exhaust gas is normally scrubbed before discharge into the atmosphere. In situ control using optical spectrometry to determine the transition between one layer and the next, or using optical interferometry to establish the depth of etching, can readily be accomplished. Selectivity of etching between two different materials is somewhat more difficult to achieve in dry etching than in wet etching, but can be very high in certain cases.

\section{Dry-etching machines}

A summary of the characteristics of six common types of dry-etching machine is shown in figure 2 . The machine types are classified by the names most often used in the literature, but the nomenclature is far from universal.

The action of the energetic ions on the substrate can be described as physical sputtering if the material is removed purely through momentum transfer: the atoms are knocked out by the impinging flux of ions. Purely physical etching is not widely used, as the etch rate tends to be slow, and the profile of an etched feature is nonvertical (see figure 3). However, it is employed in ion-beam machines (figure 2a) employing noble-gas ions. In such machines (often known as ion millers) the ions are initially created using a hot wire to ionize the gas and then are extracted by an ion gun. The resulting beam, after overall neutralization by electrons, drifts towards the target and sputters it.

In reactive etching, the gas is chosen so that ions and radicals formed after dissociation of the gas in the discharge undergo a chemical reaction with the substratepreferably one that gives a chemical product that is volatile at the process pressure so that it can be pumped away. This can be done in an ion-beam-etching machine by introducing a reactive gas in front of the substrate so that it is ionized by collision with the beam of noble-gas ions.

More usually, the discharge is formed by the action of an alternating electric field. The field accelerates any electrons, which collide with the atoms or molecules of 


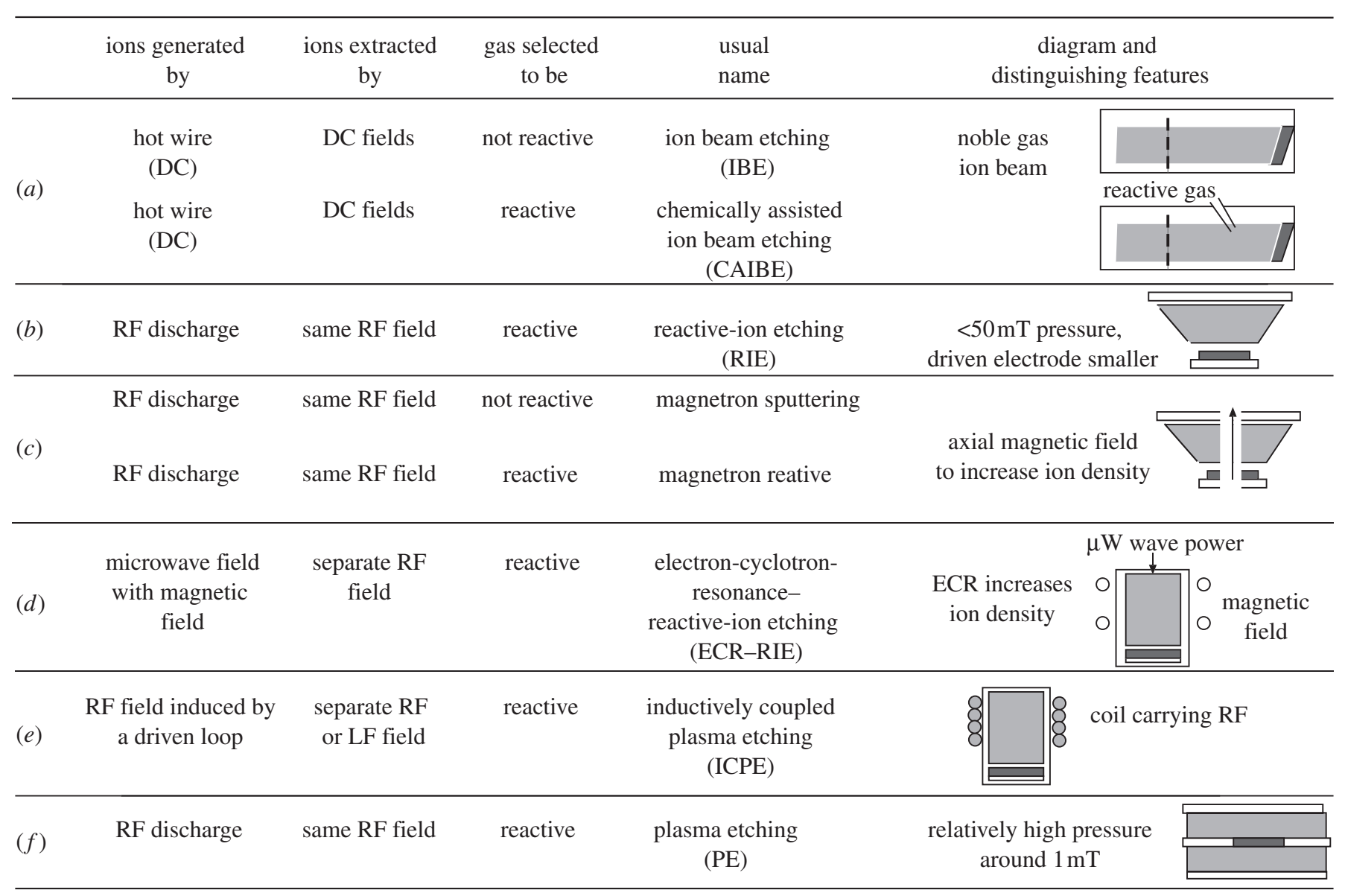

Figure 2. Classification of machine types. 


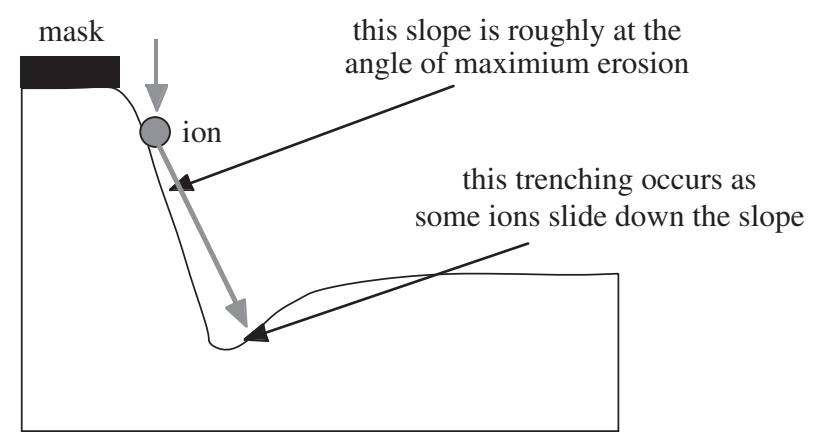

Figure 3. Typical profile of a masked opening after etching after sputtering by a non-interacting ion.

the gas, producing ions and more electrons. This process continues until a discharge is built up. Recombination of the ions into atoms or molecules by the capture or emission of an electron may be accompanied by the emission of light. The lightemitting plasma has overall electrical neutrality. At low pressures, say less than $50 \mathrm{mT}$, a dark space that is essentially free of ions appears between the electrodes and the plasma. A DC voltage is developed across this dark space, its magnitude depending on the pressure and frequency. This rectification occurs as the electrons, being of very low mass, can follow the variation of a high-frequency field, while the ions, being much heavier, may not be able to.

A reactive-ion-etching (RIE) machine (see figure $2 b$ ) is typically driven at $13.6 \mathrm{MHz}$ and at a pressure less than $50 \mathrm{mT}$. The two electrodes normally have different diameters, and the smaller one, which carries the sample to be etched, is driven by the radio-frequency ( $\mathrm{RF}$ ) field, the larger one being grounded. A voltage- the (self-) bias voltage - is developed across the dark space above the driven electrode, and the ions are accelerated across this dark space and so land upon the sample from a vertical direction. Only a small proportion of the gas molecules are ionized in an RIE machine (an ion density of $10^{8}-10^{10} \mathrm{~cm}^{-3}$ is typical (Chapman 1980)) and this proportion can be increased by increasing the RF power. Increasing the RF power also increases the bias voltage.

In a magnetron sputtering machine (see figure $2 c$ ), an axial magnetic field is applied to a machine that otherwise is similar to an RIE machine. The magnetic field causes the electrons to spiral and so increases the time they are available to ionize the gas. Thus, a more heavily ionized plasma may be obtained, and this tends to increase the etching rate at a given power level.

It can be desirable to separate the generation of the plasma from the extraction of the plasma, as this allows the density of the plasma (associated with etch rate) to be adjusted independently of the bias voltage (associated with the damage inflicted on the sample).

In an electron-cyclotron-resonance reactive-ion etching (ECR/RIE) machine (see figure $2 d$ ), the plasma is generated by a microwave field, normally at a frequency of $2.36 \mathrm{GHz}$, and extracted by an RF field at 13.6 MHz. A magnetic field (the cyclotron field $\left.B=m / e \omega_{\mathrm{C}}\right)$ of $c a .0 .08 \mathrm{~T}$ is applied axially. The effect of this field is to allow efficient transfer of energy to the electrons and so to allow efficient ionization of the gas. Such machines create relatively dense plasmas; however, the use of a low bias voltage is not a necessary guarantee of low-damage etching.

Phil. Trans. R. Soc. Lond. A (2004) 


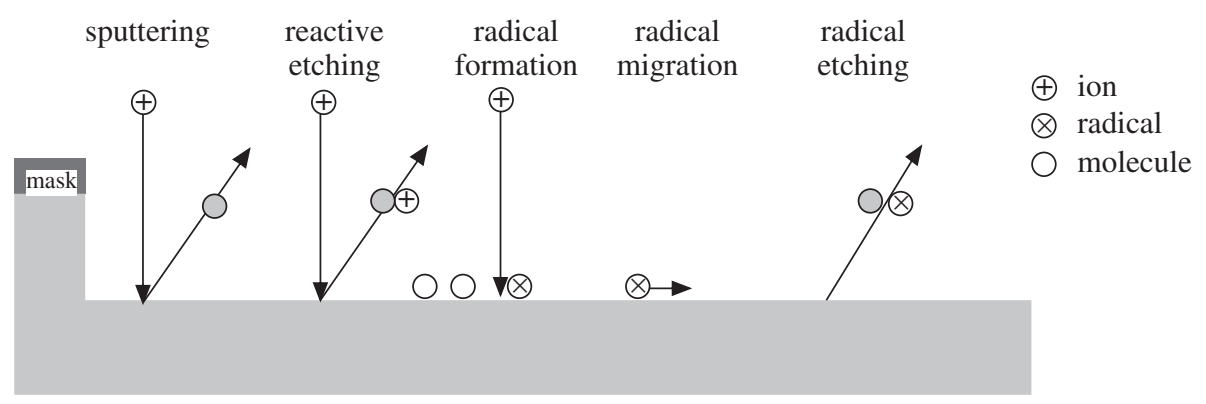

Figure 4. Processes on the surface of material during ion bombardment.

The inductively coupled plasma (ICP) etching machine (see figure $2 e$ ) is an alternative way of separating the generation of the plasma from the bias voltage. The plasma is formed by inductive coupling of RF power from an RF coil wrapped around the chamber; the ions are extracted by another RF or low-frequency supply applied to an RIE-type electrode (Layadi et al. 1999).

The plasma etching machine (see figure $2 f$ ) usually has symmetric electrodes with the sample immersed in the plasma. The self-bias voltage is low. If the etch is anisotropic, this arises from the formation of a polymeric film (from the decomposition products of the etching gas) during the process. It is widely used, with many variations in silicon processing.

\section{The role of ions in dry etching}

The term 'reactive-ion etching' is in a way, a misnomer. In an important experiment, Coburn \& Winters (1979) investigated the etching of silicon in an argon-ion beam with and without the addition of $\mathrm{XeF}_{2}$ gas injected close to the sample. They found that, while $\mathrm{XeF}_{2}$ gas by itself etched silicon slowly and the argon-ion beam by itself acted similarly, when both an ion beam and $\mathrm{XeF}_{2}$ were present the etch rate increased by a factor of more than 20 . They drew the conclusion that the ions promote the conversion of the molecules of the gas into reactive species; in particular, the ions enhance the rate of disassociative absorption of the $\mathrm{XeF}_{2}$ on the surface of the silicon wafer. It disassociates into Xe and $\mathrm{F}$ radicals and the volatile product is $\mathrm{SiF}_{4}$ (a gas at room temperature and pressure, this sublimes at $-95^{\circ} \mathrm{C}$ ). So the main role of the ions is to promote the formation of radicals from the absorbed gas in the region of the substrate that is not masked by an ion-resistant layer.

The interactions occurring on the surface of the material being etched are shown in figure 4. Some ions may sputter the surface (which is very helpful in the initial stages of etching, when inadvertent hydrocarbon contamination and surface oxide layers can be removed) and some ions may ricochet and be reflected towards the sidewalls and cause etching of them. However, the main effect of the ions is to release secondary electrons that form radicals of the absorbed gas. These radicals may immediately react with the material to be etched or they may migrate on the surface before reaction.

While the production of radicals by the ion flux will lead to a greatly enhanced rate of etching in the direction of, and over the area of, the ion flux that hits the exposed substrate, it is not clear that this is the sole reason for the highly anisotropic etching that can be obtained by RIE under the correct circumstances. Another possible cause 
of anisotropic etching is the formation of a thin etch-resistant layer on the sidewalls (sometimes called polymer formation or sidewall passivation). An example of this is found in the etching of $\mathrm{SiO}_{2}$ by $\mathrm{CHF}_{3}$. In the decomposition of $\mathrm{CHF}_{3}$, moieties containing carbon and fluorine are made, and these can give rise to a polymer similar to poly(tetrafluoroethylene). The polymer deposited on the sidewalls will sputter only slowly, while on the surface it will be removed by sputtering much more rapidly, and so vertical etching can proceed while the sidewalls are protected.

\section{Choice of gases}

The main ingredient of most etch-gas recipes is a halogen-containing gas. Fluorine is widely used with silicon and silicon compounds, and chlorine is used with Ga-, Al- and As-containing III-V compounds. However, in RIE, the volatility of indium chloride at room temperature is low, so InP and the technologically important In-containing ternary (e.g. In GaAs) and quaternary (e.g. GaInAsP) compounds require an alternative approach. Either the substrate can be heated (to more than $150{ }^{\circ} \mathrm{C}$ ) or bromineor iodine-containing gases can be used (but such gases tend to attack the vacuum system aggressively) or a mixture of methane and hydrogen can be used. The idea with methane/hydrogen is to form a hydride $\left(\mathrm{AsH}_{4}\right.$, say) and a metal-organic compound (dimethyl-gallium, say). Methane/hydrogen is a surprisingly versatile etch; introduced by Nollebrugge et al. (1985) to etch InP, it can be used for other III-V (Cheung et al. 1987) and II-VI (Foad et al. 1992) compound semiconductors and for thin-film magnetic materials (Khamsehpour et al. 1995).

In addition to the main gas, additives and dilutants are often used (Manos \& Flann 1989). Additives are used to enhance some aspect of the chemistry. For instance, the addition of $10 \% \mathrm{O}_{2}$ to $\mathrm{CF}_{4}$ increases the etch rate by 10 times. In the discharge, $\mathrm{CF}_{4}$ is broken down into radicals $\mathrm{CF}_{3}$ and $\mathrm{CF}_{2}$. In the absence of oxygen these radicals can recombine (both in the gas phase and on surfaces) into stable molecules, e.g.

$$
\mathrm{CF}_{3}+\mathrm{CF}_{3} \rightarrow \mathrm{C}_{2} \mathrm{~F}_{6} \text { and } \mathrm{CF}_{3}+\mathrm{F} \rightarrow \mathrm{CF}_{4} \text {. }
$$

With oxygen present, it can react with the radicals to form $\mathrm{CO}, \mathrm{CO}_{2}$ and $\mathrm{COF}$, thus releasing fluorine atoms that can etch

$$
\mathrm{CF}_{2}+\mathrm{O}_{2} \rightarrow \mathrm{COF}+\mathrm{F}, \quad \mathrm{COF}+\mathrm{O} \rightarrow \mathrm{CO}_{2}+\mathrm{F} .
$$

Dilutants are usually noble gases. They can stabilize the plasma. $\mathrm{SF}_{6}$ is an electronegative gas: it absorbs electrons upon ionization and so the cascade of 'an electron in, leading to a positive ion plus two electrons out' is not possible. However, the addition (and ionization) of argon adds more electrons and so helps to produce a stable plasma.

There have been two useful recent reviews of the application of dry etching to the production of Ultra Large Scale Integration integrated circuits in silicon: one from IBM (Kuo 1999) and one from Bell Laboratories (Layadi et al. 1999).

\section{Dry-etching damage}

As dry etching involves the bombardment of the semiconductor surface with energetic ions, its use brings a risk of damage to the electrical and optical properties of the semiconductor. In silicon the damage can often be annealed in a subsequent 
thermal treatment, while annealing is rarely possible in binary semiconductors, as decomposition of the material occurs at a lower temperature than would be necessary for the annealing of defects.

Dry-etching damage typically manifests itself as a surface layer of material with lower conductivity, the damaged layer being up to $100 \mathrm{~nm}$ deep. In dry-etching processes, the ions falling upon the substrate usually have energies in the range 50-500 eV. The penetration range of ions from the gases typically used in dry etching (silicon tetrachloride, fluorinated hydrocarbons, dilutants such as argon) is only a few nanometres at most for ions with energies up to $500 \mathrm{eV}$. The confused surface layer (consisting of the original semiconductor and its fragments, gas molecules and fragments of those molecules, and any involatile products of the semiconductor with the gas) has a thickness of only a few lattice spacings at most. However, it is often found that the electrical and optical properties of the semiconductor are damaged to a much greater depth.

It is generally believed that this damage is due to the introduction of traps, vacancies and interstitial atoms into the crystalline lattice. Some of these defects have been explicitly identified (Johnson et al. 1994) in the case of RIE etching of GaAs using $\mathrm{SiCl}_{4}$. These impair the electrical behaviour of the semiconductor by reducing the carrier concentration and/or the mobility and impair the optical behaviour by the introduction of recombination centres, thus reducing the photoluminescence (PL) efficiency.

The deepness of the damage was difficult to understand in the 1980s. An important observation was made by Germann et al. (1989) when he found that the damage to GaAs depended on the angle of the beam (an argon-ion beam in this case) with respect to the crystallographic axes of the specimen. This suggested something akin to channelling. Stoffel (1992) solved the problem. He used ab initio Monte Carlo calculations of the path of ions on striking the surface of a semiconductor with a diamond or zinc-blende lattice to show that, while most of the ions remained on or very close to the surface, a small fraction (ca.0.1\%) are channelled along crystallographic directions, particularly the $\langle 110\rangle$-direction for ions striking a (100) surface. These lucky ions penetrate much further into the surface and, when they dechannel at the end of their trajectory, they cause traps and vacancies as well as being new impurity atoms. This channelling occurs as the ions essentially see a more open route if they happen to be scattered at the first impact with the surface in close to one of the four $\langle 110\rangle$-directions.

The damage inflicted upon the surface of a semiconductor can be estimated in many ways.

Schottky metal-semiconductor junctions (Pang 1986) made on the damaged and untreated surface of a semiconductor show an increase in the ideality factor and decrease in the breakdown voltage but, with such measurements, it is difficult to estimate the depth of damage. In principle it is possible to wet etch a known depth into the semiconductor, but in practice this proves difficult.

Van der Pauw measurement (Knoedler et al. 1989) of an epitaxial conducting layer on top of an insulating substrate allows the characterization of the sheet conductance and mobility as a function of etch depth.

Raman scattering (Wang et al. 1992) is a useful tool for the investigation of damage, as such damage causes shifts and broadening in the phonon peaks. In particular, 
in highly doped III-V semiconductors, it allows the measurement of the depletion depth, so giving information on the depth of damage.

The method, initially introduced by Lishan et al. (1988), that we have found most useful is that of observation of PL in multiple quantum wells (QWs). A structure consisting of a series of QWs of different widths is used, each width giving PL at a particular wavelength. The PLs of etched and unetched samples are compared. The intensity of the PL in the etched samples is reduced by the defects, and, as wells are placed at a known depth from the surface, a depth profile of the damage is obtained.

The model for deep damage penetration due to low-energy dry etching is as follows. Most of the bombarding ion flux is stopped at the surface of the material being etched, with the main effect being disordering of the topmost monolayers of the crystal. However, molecular dynamics (MD) simulations in both silicon and III-V semiconductors show that, even at very low energies, $\langle 110\rangle$ ion channelling is possible (Bousetta et al. 1991). Thus, a small fraction of the order of $c a .0 .1 \%$ of the incoming ions is scattered into directions aligned with the $\langle 110\rangle$-axes. These ions channel up to tens of nanometres into the crystal and create defects when they dechannel.

We do not use MD simulations to model our experimental data, but instead exploit an analytic approximation to the damage distribution (Rahman 1995a,b). The theory we use gives an expression for the mean channelled distance, $\lambda_{c}$, which agrees with MD to within $c a .30 \%$ in the energy range up to $1 \mathrm{keV}$, with no adjustable parameters. It has been shown (Rahman et al. 1992) that, as a result of dechannelling, defects are created at a rate

$$
g(y)=\frac{\alpha J_{\mathrm{i}}}{\lambda_{\mathrm{c}}} \exp \left(-\frac{y}{\lambda}\right)
$$

where $\alpha$ is the probability of an incident ion being scattered into a $\langle 110\rangle$ channelling direction, $J_{\mathrm{i}}$ is the incident ion flux, $y$ is the depth below the surface being etched and $\lambda=\lambda_{\mathrm{c}} / \sqrt{2}$ is the mean depth in the $y$-direction.

The expression for $\lambda_{\mathrm{c}}(\mathrm{nm})$ is given below. It applies to medium mass ions only, channelling along $\langle 110\rangle$ with an initial energy up to $1 \mathrm{keV}$. We note again that there are no adjustable parameters.

$$
\begin{gathered}
\lambda_{\mathrm{c}}=4.511 \times 10^{-9} \frac{Z_{1} Z_{2}}{D_{0} a}\left(1.077 \times 10^{5} \frac{E_{0} a_{\mathrm{TF}}^{2}(f+1) D_{0} a^{3}}{Z_{1}^{2} Z_{2}^{2} u_{2}^{2}}\right)^{1 / f}, \\
D_{0}=3.729 \times 10^{-11} \frac{Z_{1}^{2}}{M_{1} a}, \quad f=2.59-0.02 \ln Z_{1} Z_{2}, \\
a_{\mathrm{TF}}=\frac{0.4693}{\left(Z_{1}^{1 / 2}+Z_{2}^{1 / 2}\right)^{2 / 3}}, \\
u_{2}=17.071 \times \sqrt{\frac{1}{M_{2} \theta_{\mathrm{m}}}\left(\frac{\phi(x)}{x}+\frac{1}{4}\right)}, \\
\phi(x)=0.9957-0.2448 x+0.0278 x^{2}-0.0012 x^{3},
\end{gathered}
$$

where $x=\theta_{\mathrm{m}} / T$ is the reduced temperature, $Z_{1}, Z_{2}, M_{1}$ and $M_{2}$ are atomic numbers and masses (AMU) of incident and target nuclei, $E_{0}$ is the forward ion energy $(\mathrm{eV})$, $a$ is the lattice constant $(\AA), T$ is the lattice temperature $(\mathrm{K})$ and $\theta_{\mathrm{m}}$ is the Debye 


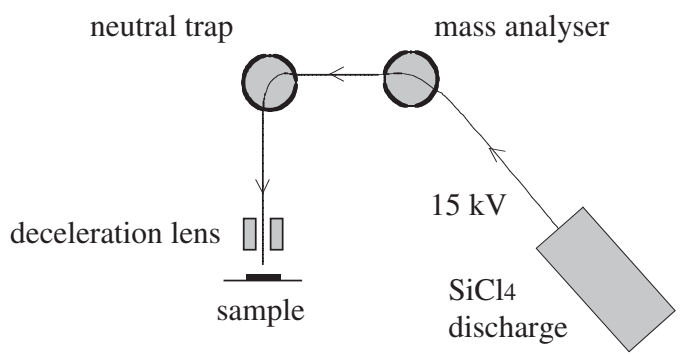

Figure 5. Schematic of a low-energy ion-implant machine.

temperature (K). For GaAs/AlGaAs we approximate $Z_{2}$ and $M_{2}$ by values for Ge, $\theta_{\mathrm{m}}$ by the Debye temperature of GaAs at $300 \mathrm{~K}$, and $a$ for $\operatorname{GaAs}\left(Z_{2}=32, \theta_{\mathrm{m}}=370 \mathrm{~K}\right.$, $a=5.63 \AA$ ). For InGaAs/InAlAs, we again use Ge values for $Z_{2}$ and $M_{2}$, but $\theta_{\mathrm{m}}$ for $\operatorname{InP}$ at $300 \mathrm{~K}$, and $a$ for $\operatorname{InP}\left(Z_{2}=32, \theta_{\mathrm{m}}=430 \mathrm{~K}, a=5.86 \AA\right)$. In our experiments $T \approx 313 \mathrm{~K}$ and we ignore possible heating of the substrate due to ion bombardment.

\section{Low-damage etching}

Extensive study has led to a number of general rules that assist in maintaining low damage. The reactive nature of the etch chemistry in RIE is important, as the enhanced etch rates lessen exposure times to the bombarding flux and remove damage as quickly as it is created. Low ion energies are essential too, to lessen the effect of channelling as described previously; it is observed from the above equation for the mean channelled depth $\lambda_{\mathrm{c}}$ that this parameter scales with the bombardment energy $E_{0}$ of the ion. However, this does not give the entire story. We observed previously that $\mathrm{SiCl}_{4}$ discharges created by ECR may sometimes lead to increased levels of damage, despite the low ion energies. This we associated with the relatively high $\mathrm{Cl}^{+}$density in the ECR discharge. To investigate the situation more thoroughly we have studied the damage due to separate $\mathrm{Si}^{+}, \mathrm{Cl}^{+},(\mathrm{SiCl})^{+},\left(\mathrm{SiCl}_{2}\right)^{+}$and $\left(\mathrm{Cl}_{2}\right)^{+}$ bombardment and due to the net bombardment in $\mathrm{a} \mathrm{SiCl}_{4} \mathrm{RIE}$ environment.

Samples were prepared using an ultra-low-energy ion implanter. A schematic of the machine is shown in figure 5. Ions are extracted from a $\mathrm{SiCl}_{4}$ discharge at $15 \mathrm{kV}$ and then are mass analysed by a bending magnet to select the desired isotope. A second bending magnet traps neutral particles and directs the ion beam towards the target. A deceleration lens just in front of the target reduces the ion energy to less than $1 \mathrm{keV}$ before bombardment. The distance between the neutral trap and target is $c a .1 \mathrm{~m}$ with the background pressure being $c a .10^{-9}$ mbar. Energy contamination of the ion beam due to $15 \mathrm{keV}$ neutrals passing unaffected through the deceleration lens is at the level of less than $0.01 \%$. Figure $2 b$ shows the typical RIE set-up. Ions bombard the sample in both RIE and the implanter. In the case of RIE, a mixture of many types of ion is directed towards the sample; in the case of the implanter single species of ion may be selected.

The implant machine was used to implant ions into GaAs/AlGaAs QW materials at energies of up to $300 \mathrm{eV}$ and flux densities of $10^{13}-10^{14}$ ions $\mathrm{cm}^{-2} \mathrm{~s}$, comparable with the conditions experienced inside an RIE chamber. The atomic ions $\mathrm{Si}^{+}$and ${ }^{35} \mathrm{Cl}^{+}$and the molecular ions $(\mathrm{SiCl})^{+},\left(\mathrm{SiCl}_{2}\right)^{+}$and $\left(\mathrm{Cl}_{2}\right)^{+}$were selected. Regions of the samples were masked, as described already, to allow measurement of PL from 

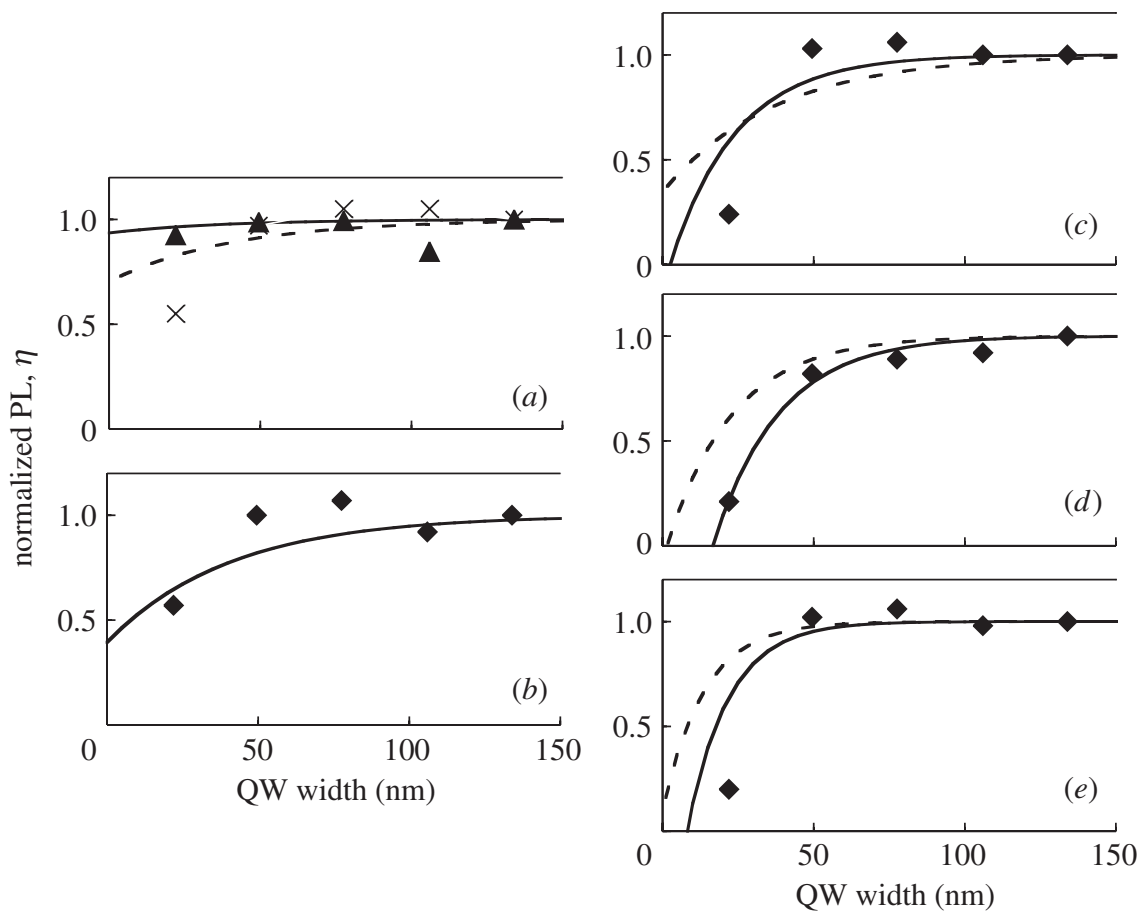

Figure 6. Experimental PL measured after bombardment from atomic and molecular ions in the implanter. Lines denote theoretical values. (a) $\mathrm{Cl}^{+}$implanted for $10 \mathrm{~s}$ (triangles) and $50 \mathrm{~s}$ (crosses) at $300 \mathrm{eV} .(b) \mathrm{Si}^{+}$implanted for $75 \mathrm{~s}$ at $300 \mathrm{eV} .(c)(\mathrm{SiCl})^{+}$implanted for $325 \mathrm{~s}$, theory lines are for $300 \mathrm{eV} \mathrm{Cl}^{+}$(dashed line) and $80 \mathrm{eV} \mathrm{Cl}^{+}$(solid line). (d) $\left(\mathrm{SiCl}_{2}\right)^{+}$implanted for $515 \mathrm{~s}$, theory at single $\mathrm{Cl}^{+}$flux (dashed line) and double $\mathrm{Cl}^{+}$flux (solid line) at $300 \mathrm{eV} .(e)\left(\mathrm{Cl}_{2}\right)^{+}$ implanted for $350 \mathrm{~s}$ at $100 \mathrm{eV}$, theory at single $\mathrm{Cl}^{+}$flux (dashed line) and double $\mathrm{Cl}^{+}$flux (solid line).

unexposed QWs. Talystep measurements confirmed any etching to be less than $10 \mathrm{~nm}$ deep.

A second group of GaAs/AlGaAs samples was etched in $\mathrm{SiCl}_{4} \mathrm{RIE}$ using a $12 \mathrm{~W}$ process (Deng et al. 2000). Both RIE and ion-implanted samples were measured using PL to probe the damage profile.

The model of damage is most readily applied to samples exposed within the ion implanter, which we discuss first. The data points in figure $6 a$ show the normalized PL measured from samples exposed to $300 \mathrm{eV} \mathrm{Cl}^{+}$in the ion implanter for two different exposure times and doses $\left(10 \mathrm{~s}\right.$, ion dose of $10^{15}$ ions $\mathrm{cm}^{-2}$ and $50 \mathrm{~s}, 5 \times$ $10^{15}$ ions $\mathrm{cm}^{-2}$, respectively). The lines are calculations based on the model described earlier. An increased dose leads to the accumulation of damage, as expected, because the etch rate is low.

Figure $6 b$ shows photoluminescence from samples exposed to $300 \mathrm{eV} \mathrm{Si}^{+}$at a dose of $10^{16}$ ions $\mathrm{cm}^{-2}$. At this energy, we observe that $\mathrm{Si}^{+}$reduces the PL intensity of the first QW. Again, the lines denote calculations based on the model described above. For both $\mathrm{Cl}^{+}$and $\mathrm{Si}^{+}$the model calculations are in reasonable agreement with measured data. Previous experiments using the ion implanter suggest that at 
very much lower energies $(c a .50 \mathrm{eV}) \mathrm{Si}^{+}$does not channel, but prefers instead to deposit on GaAs.

The model described already applies only to atomic ions. An estimate of the atomic-ion radius is given by the Thomas-Fermi length, $a_{\mathrm{TF}} \approx 0.01 \mathrm{~nm}$. In contrast, molecular ions are much larger, due to the bond length, e.g. $c a .0 .20 \mathrm{~nm}$ for $\mathrm{Si}-\mathrm{Cl}$. Comparing this with the channel diameter, ca. $0.26 \mathrm{~nm}$ for $\langle 110\rangle \mathrm{GaAs}$, we see that the irregular shape and tumbling motion of molecular ions as they pass by lattice nuclei ensures rapid large-angle scattering before channelling can begin. Such molecular ions do not experience the smooth channel potential felt by atomic ions and so cannot penetrate to any significant depth along low-index axes.

However, molecular ions may fragment into atomic ions upon impact at the surface. These secondary atomic ions may channel, but at an energy much less that that of the incident molecular ion. The situation is analogous to what happens when creating ultra-shallow junctions in silicon by high-energy implantation of large molecular ions. For example, in $30 \mathrm{kV}$ implantation of $\mathrm{BF}_{2}$, the $\mathrm{BF}_{2}$ molecules fragment upon impacting on the silicon surface and much lower energy boron implants into the material (Smith et al. 1998).

Having studied atomic-ion bombardment in the implanter, we continued to examine the effect of molecular ion bombardment. Figure $6 c, d$ shows the effect of exposure of the GaAs/AlGaAs QW layers to the molecular ions $(\mathrm{SiCl})^{+}$and $\left(\mathrm{SiCl}_{2}\right)^{+}$, at an energy of $300 \mathrm{eV}$ and ion dose of $10^{16}$ ions $\mathrm{cm}^{-2}$. Figure $6 e$ shows the effect of exposure to $\left(\mathrm{Cl}_{2}\right)^{+}$at an energy $100 \mathrm{eV}$ and ion dose of $10^{15}$ ions $\mathrm{cm}^{-2}$.

We have argued that molecular ions do not channel, but fragmentation upon impact may give lower energy atomic ions that may channel. As mentioned earlier, at very low energies $\mathrm{Si}^{+}$has been observed to accumulate so we assume that this causes no significant measurable damage. Hence, the calculations plotted in figure $6 c-e$ are for $\mathrm{Cl}^{+}$at energies much lower than the incident molecular-ion energy. The calculations allow for generation of $\mathrm{Cl}^{+}$at twice the dose of $\left(\mathrm{SiCl}_{2}\right)^{+}$and $\left(\mathrm{Cl}_{2}\right)^{+}$, as these have twice as many $\mathrm{Cl}$ nuclei. We chose a secondary ion energy that is $c a .25 \%$ of the incident molecular ion energy, to allow for kinematic effects and energy loss during impact. From figure $6 c$ it is clear that the low-energy $\mathrm{Cl}^{+}$flux describes the experimental data well, and that in all cases (figure $6 c-e$ ) the effect of fragmentation is evident in enhancing the secondary $\mathrm{Cl}^{+}$ion flux.

We conclude that the model is able to describe well the effects of both atomic and molecular ion bombardment. This permits the analysis of the more complex situation arising within RIE machines, where the bombarding ion flux is a mixture of many types of ion.

Thus, for comparison with the implanter data, the GaAs/AlGaAs was also etched using $\mathrm{SiCl}_{4} \mathrm{RIE}$ to test for the effect of the net bombardment. When very low power $\mathrm{SiCl}_{4} \mathrm{RIE}$ was used (10 W DC self-bias $c a .35 \mathrm{~V}$ ), with the flow rate and pressure given previously, no significant deterioration in PL intensity was evident even after 5 min of etching time. This showed that the etch stop works and that any subsequent oxidation of the surface exposed to air does not affect the PL of the topmost QW.

A sample was also etched at $12 \mathrm{~W}$ (DC self-bias $80 \mathrm{~V}$ ) with the $\mathrm{SiCl}_{4}$ process described already. The data points of figure 7 show the resulting PL. The discharge contains a mixture of $\mathrm{Si}^{+}, \mathrm{Cl}^{+},\left(\mathrm{SiCl}_{x}\right)^{+}$, and $\left(\mathrm{Cl}_{2}\right)^{+}$according to optical-emission spectroscopy, together with various neutrals. Although the exact composition of the bombarding ion flux is not known, it will comprise some combination of the above 


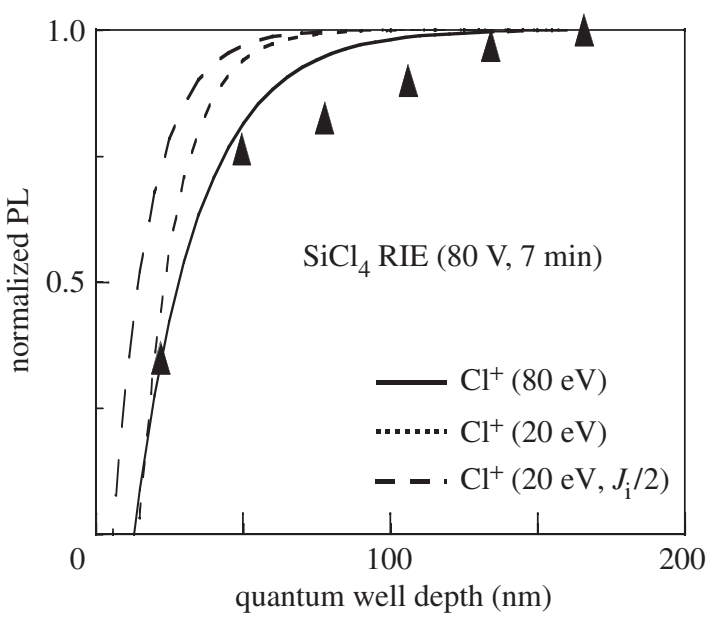

Figure 7. Experimental PL measured after exposure of the sample within an RIE machine.

ions. We have said that the bombarding molecular ions do not channel, but will create secondary atomic ions that do, although only with energy much less than $80 \mathrm{eV}$. We have also said that at these low energies $\mathrm{Si}^{+}$does not produce significant observable damage.

Figure 7 also shows theoretical curves calculated for $80 \mathrm{eV}$ and $20 \mathrm{eV} \mathrm{Cl}^{+}$. This allows a comparison of the effect expected for atomic $\mathrm{Cl}^{+}$bombardment of the sample surface directly from the discharge $(80 \mathrm{eV})$, or $\mathrm{Cl}^{+}$channelling as a result of a molecular ion impact $(20 \mathrm{eV})$. The model curves show that the higher energy $(80 \mathrm{eV})$ ions contribute more to deep damage. The agreement with experiment suggests that the damage occurs primarily from atomic $\mathrm{Cl}^{+}$ions direct from the RIE discharge. The data obtained for more complex discharges further support the conclusion that the PL degradation as a result of etching may be explained primarily in terms of atomic-ion channelling alone (Rahman et al. 2001).

This would explain why we have observed enhanced levels of damage in $\mathrm{SiCl}_{4}$ ECR as compared to that in $\mathrm{SiCl}_{4}$ RIE: increased atomic $\mathrm{Cl}^{+}$generation in ECR as observed using optical emission spectroscopy leads to more significant levels of deep damage penetration. Presumably a similar conclusion holds for all high-density etch systems, including ICP, although we have not tested this explicitly. This means that, when etching nanostructures using either ECR or ICP, one should avoid conditions that create a large density of atomic ions in the discharge, as this will raise levels of damage above the minimum possible value even if quite low ion energies are being used.

\section{Conclusions}

Dry etching plays a fundamental role in the fabrication of semiconducting devices. Much development worldwide has gone into the realization of efficient processes that allow reliable etching of nanometre-sized features of the desired profile.

Ion sputtering plays a vital but subtle role in dry etching. On the one hand, the use of sputtering to remove material by momentum transfer is normally avoided in practical dry-etching processes; as the etch rate is low and the profile inevitably nonvertical. On the other hand, the energy release at the surface being bombarded by the 
ions crucially gives rise to the formation of the radicals that are the principal cause of removal of material during dry etching. The kinetic energy of the bombarding ions can give rise to the penetration of the ions into the material, and some of these ions can be channelled along crystallographic directions to considerable depth. However, in III-V semiconductors, this potential source of optical and electrical damage can be minimized by a suitable choice of conditions that avoid the presence of atomic ions and rely on molecular ions to undertake the bulk of the etching.

The work reviewed in this paper has benefited from the collaboration of many colleagues. In particular this includes Rebecca Cheung, Majeed Foad, Li-Gang Deng, Saad Murad, Clivia Sotomayor Torres, Steve Thoms, Steve Beaumont, Colin Stanley, Gordon Doughty and Nigel Johnson at the University of Glasgow, and Jaap van den Berg and Dave Armour at the University of Salford and Evelyn Hu at the University of California Santa Barbara. The technical assistance of Dave Clifton, Colin Roberts and Ronnie Roger in the Dry Etching Facility at the University of Glasgow is gratefully acknowledged.

\section{References}

Bousetta, A., van den Berg, J. A., Valizedah, R., Armour, D. G. \& Zalm, P. C. 1991 Ultra low energy (100-2000 eV) boron implantation into crystalline and silicon-preamorphized silicon. Nucl. Instrum. Meth. Phys. Res. B 55, 565.

Chapman, B. N. 1980 Glow discharge processes: sputtering and plasma etching. Wiley.

Cheung, R., Thoms, S., Beaumont, S. P., Doughty, G., Law, V. \& Wilkinson, C. D. W. 1987 Reactive ion etching of GaAs using a mixture of methane and hydrogen. Electron. Lett. 23, 857-859.

Coburn, J. W. \& Winters, H. F. 1979 Ion-assisted and electron-assisted gas-surface chemistry: important effects in plasma-etching. J. Appl. Phys. 50, 3189-3196.

Deng, L. G., Rahman, M. \& Wilkinson, C. D. W. 2000 Enhanced damage due to light in lowdamage reactive-ion etching processes. Appl. Phys. Lett. 76, 2871-2873.

Foad, M. A., Wilkinson, C. D. W., Dunscomb, C. \& Williams, R. H. $1992 \mathrm{CH}_{4} / \mathrm{H}_{2}$ - a universal reactive ion etch for II-VI semiconductors. Appl. Phys. Lett. 60, 2531-2533.

Germann, R., Forchel, A., Bresch, M. \& Meier, H. P. 1989 Energy-dependence and depth distribution of dry etching-induced damage in III/V semiconductor heterostructures. J. Vac. Sci. Technol. B 7, 1475-1478.

Johnson, N. P., Foad, M. A., Murad, S., Holland, M. C. \& Wilkinson, C. D. W. 1994 Deep levels induced by $\mathrm{SiCl}_{4}$ reactive ion etching in GaAs. Mater. Res. Soc. Symp. Proc. 325, 443-448.

Khamsehpour, B., Wilkinson, C. D. W. \& Chapman, J. N. 1995 Fabrication of NiFe thin-film elements by dry-etching using $\mathrm{CH}_{4} / \mathrm{H}_{2} / \mathrm{O}_{2}$. Appl. Phys. Lett. 67, 3194-3196.

Knoedler, C. M., Osterling, L. \& Heiblum, H. 1989 Inert-gas reactive ion etching damage to GaAs using inverted heterojunctions. J. Appl. Phys. 65, 1800-1802.

Kuo, Y. (ed.) 1999 Plasma processing. IBM J. Res. Develop. 43, 1-215.

Layadi, N., Colonell, J. I. \& Lee, J. T.-C. 1999 An introduction to plasma etching for VLSI circuit technology. Bell Labs Tech. J. 4, 155.

Lishan, D. G., Wong, H. F., Green, D. L., Hu, E. L. \& Merz, J. L. 1988 Dry etch induced damage in GaAs investigated using Raman-scattering spectroscopy. J. Vac. Sci. Technol. B 7, 556-560.

Manos, D. N. \& Flann, D. L. 1989 Plasma etching-an introduction. Academic.

Nollebrugge, U., Klug, M. \& Gardus, G. 1985 A novel process for reactive ion etching in InP using $\mathrm{CH}_{4} / \mathrm{H}_{2}$. In Gallium Arsenide and Related Compounds 1985. Proc. 12th Int. Symp. on Gallium Arsenide and Related Compounds, Karuizawa, Japan, 23-26 September 1985 (ed. M. Fujimoto). Institute of Physics Conference Series, vol. 79, pp. 367-387. Bristol: Institute of Physics Publishing.

Phil. Trans. R. Soc. Lond. A (2004) 
Pang, S. W. 1986 Surface damage on GaAs induced by reactive ion etching and sputter etching. J. Electrochem. Soc. 133, 784-787.

Rahman, M. 1995 a Channelling and diffusion in dry-etch damage. J. Appl. Phys. 82, 2215-2224.

Rahman, M. $1995 b$ Quantum heat bath theory of dechannelling. Phys. Rev. B 52, 3383-3389.

Rahman, M., Johnson, N. P., Foad, M. A., Long, A. R., Holland, M. C. \& Wilkinson, C. D. W. 1992 Model for conductance in dry-etch damaged N-GaAs structures. Appl. Phys. Lett. 61, 2335-2337.

Rahman, M., Deng, L. G., Wilkinson, C. D. W. \& van den Berg, J. A. 2001 Studies of damage in low-power reactive-ion etching of III-V semiconductors. J. Appl. Phys. 89, 2096-2108.

Smith, R., Shaw, M., Webb, R. P. \& Foad, M. A. 1998 Ultrashallow junctions in Si using decaborane? A molecular dynamics simulation study. J. Appl. Phys. 83, 3148-3152.

Stoffel, N. G. 1992 Molecular-dynamics simulations of deep penetration by channelled ions during low-energy ion-bombardment of III-V semiconductors. J. Vac. Sci. Technol. B 10, 651-658.

Wang, P. D., Foad, M. A., Sotomayor-Torres, C. M., Thoms, S., Watt, M., Cheung, R., Wilkinson, C. D. W. \& Beaumont, S. P. 1992 Raman-scattering of coupled longitudinal optical phonon-plasmon modes in dry etched $n^{+}$-GaAs. J. Appl. Phys. 71, 3754-3759.

Phil. Trans. R. Soc. Lond. A (2004) 